\section{LONG-TERM DAY-AND-NIGHT ROTATING SHIFT WORK POSES A BARRIER AGAINST THE NORMALISATION OF LIVER FUNCTION}

1,2Yu-Cheng Lin, ${ }^{3}$ I-Chun Hsieh, ${ }^{2}$ Pau-Chung Chen. ${ }^{1}$ En Chu Kong Hospital, Department of Occupational Medicine, New Taipei, Taiwan; ${ }^{2}$ Institute of Occupational Medicine and Industrial Hygiene, College of Public Health, National Taiwan University, Taipei, Taiwan; ${ }^{3}$ Taiwan Adventist Hospital, Environmental and Occupational Medicine, Taipei, Taiwan

\subsection{6/oemed-2014-102362.223}

Objectives To evaluate the impact of day-and-night rotating shift work (RSW) on liver health, we analysed the association between long term RSW exposure and the normalisation of plasma alanine transaminase (ALT) levels over a five-year period. Method The data from physical examinations, blood tests, abdominal sonographic examinations, personal histories, and occupational records were collected from a cohort of workers in a semiconductor manufacturing company. The sample population was divided into three subgroups for analysis: persistent daytime workers, workers exposed intermittently to RSW (iRSW), and exposed to persistent RSW (p-RSW).

Results Records were analysed for 1196 male workers with an initial mean age of 32.5 years (SD 6.0 years), of whom 821 were identified as rotating shift workers, including 374 i-RSW and 447 p-RSW workers. At the beginning of the follow-up, 275 were found to have elevated ALT (e-ALT): $25.1 \%$ day-time workers, $23.0 \%$ i-RSW workers and $21.3 \%$ p-RSW workers. Of those with e-ALT at the beginning, 101 workers showed normalised serum ALT levels at the end of five-year follow-up: 10.7\% of day-time workers, $8.6 \%$ of i-RSW workers, and $6.5 \%$ of pRSW workers; $\mathrm{P}=0.016$ ). By performing multivariate logistic regression analyses, and comparing with the persistent daytime co-workers, after controlling for confounding variables, analysis indicated that the workers exposed to p-RSW were $46 \%$ less likely (OR, $0.54 ; 95 \% \mathrm{CI}, 0.30-0.95 ; \mathrm{P}=0.03$ ) to attain normal ALT levels within a five-year interval.

Conclusions Persistent day-and-night RSW pose a vigorous obstacle to the normalisation of e-ALT among workers with preexing abnormal liver function.

\section{RECOVERY FROM MENTAL CONDITION: IS IT DIFFERENT BETWEEN TBI/NON-TBI}

${ }^{1}$ Kuan-Han Lin, ${ }^{2} J u d i t h$ Shu-Chu Shiao, ${ }^{3}$ Shih-Cheng Liao, ${ }^{4}$ Chun-Ya Kuo, ${ }^{5}$ Yue Leon Guo, ${ }^{6}$ Nai-Wen Guo. ${ }^{1}$ Department of Social Medicine, College of Medicine, National Taiwan University, Taipei, Taiwan; ${ }^{2}$ Department of Nursing, College of Medicine, National Taiwan University (NTU) and NTU Hospital, Taipei, Taiwan; ${ }^{3}$ Department of Psychiatry, National Taiwan University Hospital, Taipei, Taiwan; ${ }^{4}$ Department of Psychiatry, Chung Shan Medical University Hospital, Taichung, Taiwan; ${ }^{5}$ Department of Environmental and Occupational Medicine, National Taiwan University Hospital, Taipei, Taiwan; ${ }^{6}$ Institute of Behavioral Medicine, National Cheng Kung University, Tainan, Taiwan

\subsection{6/oemed-2014-102362.224}

Objectives This study aimed to determine the rates of psychological symptoms among those with traumatic brain injury (TBI) and with non-TBI at 3 months and 12 months after occupational injury and to examine the change in psychological status over time.

Method Our study candidates were injured workers in Taiwan who were hospitalised for 3 days or longer and received hospitalisation benefits from the Labour Insurance. A self-reported questionnaire including Brief Symptom Rating Scale (BSRS-50) and Post-traumatic Symptom Checklist (PTSC) was sent to workers at 3 months and 12 months.

Results Among 853 injured workers who completed the questionnaire at 3 and 12 months, regarding to the severity of BSRS score, $7.8 \%$ of those with TBI had recovered at 12 months, comparing with $8.1 \%$ in those with non-TBI. On the other hand, approximately $11.6 \%$ of those with TBI had recovered from post-traumatic stress symptoms at 12 months, comparing with $9.7 \%$ among those with non-TBI. Injured workers with TBI had lower rate of recovery from psychological symptoms, comparing with non-TBI.

Conclusions A significant proportion of victims with TBI and non-TBI suffered psychological symptoms after injury. The identification and treatment of psychological symptoms are important for optimal adaptation after traumatic injury.

\section{OCCUPATIONAL DEAFNESS DUE TO CO-EXPOSURE TO NOISE AND OTOTOXIC AGENTS}

Zoubida Belhadj, Chahrazed Kandouci, Baderdine Abdelkrim Kandouci. UDL Sidi Bel Abbes, Sidi Bel Abbes, Algeria

10.1136/oemed-2014-102362.225

Objectives In order to estimate the hearing impairment in occupational environment due to the co-exposure to noise and solvents, we have undertaken a cross-sectional study to evaluating the prevalence of hearing loss due the co-exposure to both solvents and noise.

Method We recruited 144 workers, working in electronic materials manufacturing plant, one group is exposed to solvents alone, and the other one exposed to both noise and solvents. In another hand, we followed two other groups in a construction company of farm implements. 136 workers exposed to noise, 96 administrative workers none exposed. The data were collected by means of questionnaire and of an introductory tonal audiometry between 0,125 and $8 \mathrm{KHz}$

Results The prevalence of hearing loss of more $20 \mathrm{~dB}$ in the group exposed to the noise and solvents was mush greater $(57$. $8 \%$ ) than that of the noise alone (35.3\%), and that of administrative workers $(27.7 \%)(\mathrm{P}<0.0001)$.

Multivariate logistic regression analysis showed that the solvents and noise group had an estimated risk for hearing loss $>20$ $\mathrm{dB}$ about 4.4 times higher than that of the noise group.

Hearing impairment was greater for speech frequency than for high frequency.

Conclusions Our results suggest that solvents increase potentially the hearing loss in a noisy environment, with a higher impact on the speech frequencies.

\section{CHANGES IN VENTILATORY AND HAEMODYNAMIC PARAMETERS DURING EXPOSURE TO ULTRAFINE PARTICLES IN A MANUFACTURING FARM MACHINERY}

Zoubida Belhadj, Chahrazed Kandouci, Baderdine Abdelkrim Kandouci, Faiza Belmokhtar. UDL Sidi Bel Abbes, Sidi Bel Abbes, Algeria

10.1136/oemed-2014-102362.226

Objectives To evaluate the influence of occupational exposure to ultrafine particles on cardiopulmonary parameters. 